This report was prepared as an account of work sponsored by an agency of the United States Government. Neither the Unired States Government not any agency thereof, nor any of their employees, makes any warranty, express or implied, or assumes any legal liability or responsibility for the accuracy, compleseness, or usefuiness of any information, apparatus, product, or process disclosed, of represents that its use would not infringe privately owned rights. Reference herein to any specific commercial product, process, or service by trade aame, trademark, manufac: turer, or otherwise does not necessariily constitute or imply its endorsement, recommendation, or favoring by the United States Govermment or any agency thereof. The views and opinions of authors expressed herein do not necessarily state or reflect those of the United States Government or any agency thereof. 
do not work for charged-particle beams. It is hoped, however, that the methods presented here provide some progress toward a multidimensional, deterministic electron-transport capability.

Coupled electron-photon transport capability is needed for an ever-increasing number of applications, including the response of electronics components to space and man-made radiation environments, medical radiation therapy, industrial curing, and food sterilization. Currently, powerful Monte Carlo codes are available for electron transport ${ }^{4}$. While some problems are well suited for a Monte-Carlo approach, other problems are more efficiently solved with a deterministic method, e.g. computing distributions, modeling deep penetration, and low-probability events.

Deterministic electron transport capability has been available for one-dimensional geometries, with the CEPXS/ONELD code package ${ }^{5}$. Unfortunately, the CEPXS/ONELD approach is not easily extendable to multidimensional geometries. Bill Filippone and his students at the University of Arizona developed the multidimensional, deterministic charged-particle code, SMARTEPANTS ${ }^{6}$. This code is presently under active development.

The work presented here combines the CEPXS/ONELD and SMARTEPANTS approaches, resulting in SMARTEPANTS-like cross sections that are compatible with production discreteordinates codes. The resulting cross sections have a number of desirable properties: 1) positivity, 2) much smaller than the true interaction cross sections, 3) low-order Legendre expansion, 4) and not tied to a particular quadrature set. These desirable properties will be further explained, with limitations given, later in this article.

\section{BOLTZMANN-FOKKER-PLANCK OPERATOR}

The Boltzmann operator, $\mathrm{L}$, describing the distribution in space, direction, and energy of a field of either charged or neutral particles is defined by ${ }^{7}$

$$
\begin{gathered}
\mathbf{L} \Phi(\mathbf{r}, \boldsymbol{\Omega}, E)=-\boldsymbol{\Omega} \bullet \nabla \Phi(\mathbf{r}, \boldsymbol{\Omega}, E)-\sigma_{t}(\mathbf{r}, E) \Phi(\mathbf{r}, \boldsymbol{\Omega}, E) \\
\quad+\iint \sigma_{s}\left(\mathbf{r}, \boldsymbol{\Omega}^{\prime} \rightarrow \boldsymbol{\Omega}, E^{\prime} \rightarrow E\right) \Phi\left(\mathbf{r}, \boldsymbol{\Omega}^{\prime}, E^{\prime}\right) d \boldsymbol{\Omega}^{\prime} d E^{\prime}
\end{gathered}
$$

The scattering cross section, $\sigma_{s}\left(\mathbf{r}, \boldsymbol{\Omega}^{\prime} \rightarrow \Omega, E^{\prime} \rightarrow E\right)$, is the probability per unit energy and solid angle that a particle of energy $E^{\prime}$ moving in direction $\Omega^{\prime}$ will be scattered to energy $E$ in direction $\boldsymbol{\Omega}$. The total cross section is $\sigma_{t}(\mathbf{r}, E)$. For neutral-particle applications, the Boltzmann equation is normally solved by expanding the scattering cross section in a low-order Legendre polynomial expansion and the discretizing the spatial, energy, and angular dependence of the fluence.

For scattering that is highly forward peaked, which is characteristic of charged-particle scattering, the Legendre polynomial angular expansion of the cross sections that is normally used in discrete ordinates codes is inadequate. The Boltzmann-Fokker-Planck (BFP) operator is an approximation to the Boltzmann-transport operator for scattering interactions that are highly forward peaked.

In order to model electron transport with a BFP formulation, the scattering cross section is separated into three components, 1) the elastic-scattering part (for directional change without energy 
loss), 2) a soft inelastic-scattering part (for energy loss without significant directional change), and 3) a hard inelastic-scattering part (for both energy loss and directional change). The soft-inelastic portion of the scattering cross section is approximated by Continuous-Slowing-Down (CSD) theory, so that the Boltzmann-Fokker-Planck operator is ${ }^{8}$

$$
\begin{aligned}
& \mathbf{L}_{B F P} \Phi(\mathbf{r}, \boldsymbol{\Omega}, E)=-\boldsymbol{\Omega} \bullet \nabla \Phi(\mathbf{r}, \boldsymbol{\Omega}, E)-\left(\sigma_{e l}+\sigma_{i n}^{h}\right) \Phi(\mathbf{r}, \boldsymbol{\Omega}, E)+\frac{\partial}{\partial E}[S \Phi] \\
& +\int \sigma_{e l}\left(\mathbf{r}, \mu_{0}, E\right) \Phi\left(\mathbf{r}, \boldsymbol{\Omega}^{\prime}, E\right) d \boldsymbol{\Omega}^{\prime}+\iint \sigma_{i n}^{h}\left(\mathbf{r}, \mu_{\mathbf{0}}, E^{\prime} \rightarrow E\right) \Phi\left(\mathbf{r}, \boldsymbol{\Omega}^{\prime}, E^{\prime}\right) d \boldsymbol{\Omega}^{\prime} d E^{\prime}
\end{aligned}
$$

where the stopping power is defined by

$$
S(\mathbf{r}, E)=\int_{E}^{E+\delta} d E^{\prime} \sigma_{i n}^{s}\left(\mathbf{r}, E^{\prime} \rightarrow E\right)\left(E-E^{\prime}\right),
$$

where $\delta$ is the energy boundary (artificial) between soft and hard scattering.

The inelastic-scattering cross section is separated into two components by specifying an artificial energy and directional boundary separating the two components. For energy loss greater than the artificial boundary value, the inelastic-scattering cross section is handled with a standard multigroup-Legendre expansion, requiring that this part of the cross section not be too anisotropic. For energy loss less than the artificial boundary, the inelastic-scattering cross section is modeled by the continuous-slowing-down theory, requiring that the angular change be negligible. In the CEPXS code $^{5}$, down scattering to an adjacent group is treated with CSD theory and, down scattering beyond an adjacent group is treated with the standard multigroup-Legendre expansion.

\section{THE GOUDSMIT-SAUNDERSON OPERATOR}

The Goudsmit-Saunderson operator ${ }^{9}$ includes 1) the elastic-scattering part (for directional change without energy loss), and 2) the soft inelastic-scattering part (for energy loss without significant directional change), and neglects 3 ) the hard inelastic-scattering part (for both energy loss and directional change). The Goudsmit-Saunderson formulation solves the infinite-medium problem. The value of the Goudsmit-Saunderson equation is that it can be solved exactly. The Goudsmit-Saunderson operator is

$$
\mathbf{L}_{G S} \Phi(\boldsymbol{\Omega}, E)=-\sigma_{e l} \Phi(\boldsymbol{\Omega}, E)+\int \sigma_{e l}\left(\mu_{0}, E\right) \Phi\left(\boldsymbol{\Omega}^{\prime}, E\right) d \boldsymbol{\Omega}^{\prime}+\frac{\partial}{\partial E}[S \Phi]
$$

The Goudsmit-Saunderson solution is based upon the expansion of the angular fluence and elastic-scattering cross section in infinite Legendre-polynomial expansions. The elastic-scattering cross section is extremely forward peaked and, therefore, requires a huge number of Legendre expansion coefficients for accurate modeling (typically $\sim 200$ ). If the angular fluence and the elastic scattering cross section are expanded in infinite Legendre-polynomial series, the source-free Goudsmit-Saunderson equation becomes

$$
\sum_{n=0}^{\infty} \frac{2 n+1}{2} P_{n}(\mu)\left[\frac{d}{d E}\left[S \phi^{n}\right]-\left(\sigma_{e l}^{0}-\sigma_{e l}^{n}\right) \phi^{n}\right]=0
$$


where $\phi^{n}$ and $\sigma_{e l}^{n}$ are the Legendre moments of the fluence and elastic-scattering cross section, respectively.

Multiplying by $P_{n^{\prime}}(\mu)$, integrating over $\mu$, and integrating from energy $E$ to energy an upperenergy bound $E_{0}$, the angular fluence is

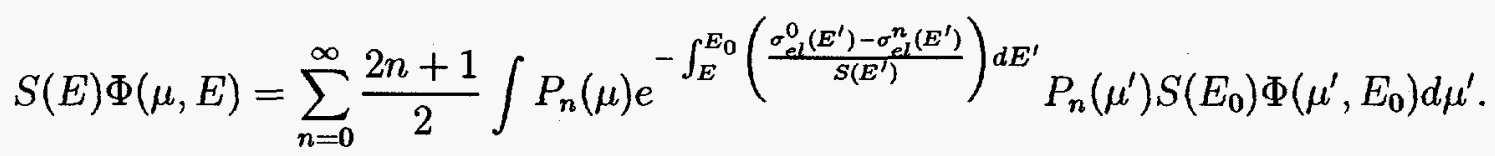

Eq. (6) is the basis for computing SMART multigroup-Legendre cross sections. The success of the method depends on: 1) accurately relating the boundary fluences to the multigroup fluences, and 2) the exponential term introducing enough anisotropy into the scattering such that the Legendre expansion converges with a small number of terms.

\section{MULTIGROUP-LEGENDRE CROSS SECTIONS}

The multigroup-Legendre form of the Goudsmit-Saunderson equation is

$$
\sigma_{t, g} \Phi_{g}(\mu) \cong \sum_{l=0}^{L} \frac{2 l+1}{2} \int P_{l}(\mu) \sigma_{g^{\prime} \rightarrow g}^{l} P_{l}\left(\mu^{\prime}\right) \Phi_{g^{\prime}}\left(\mu^{\prime}\right) d \mu^{\prime}
$$

There are a number of possible ways to relate Eqs. (6) and (7). The manner in which this is done has a profound effect on the resulting cross sections, and we present two methods here. The first method results in cross sections that are all positive, which is certainly a desirable property. The second method results in cross sections that may be negative, but results in more accurate solutions.

\section{A. Method 1: Relate boundary fluence to adjacent multigroup fluences}

Integrating Eq. (6) over energy group $\mathrm{g}+1$ and dividing by the group width results in

$$
\begin{gathered}
\frac{S_{g+1} \phi_{g+1}(\mu)}{\Delta E}=\int\left\{\frac{1}{2}+\sum_{n=1}^{\infty}\left(\frac{2 n+1}{2}\right) P_{n}(\mu)\left(\frac{S_{g+1} / \Delta E}{\sigma_{g+1}^{0}-\sigma_{g+1}^{n}}\right)\right. \\
\left.\left[1-e^{-\left(\frac{\sigma_{g+1}^{0}-\sigma_{g+1}^{n}}{S_{g+1}}\right) \Delta E}\right] P_{n}\left(\mu^{\prime}\right)\right\} S_{g+\frac{1}{2}} \phi\left(\mu^{\prime}, E_{g+\frac{1}{2}}\right) d \mu^{\prime}
\end{gathered}
$$

where the upper energy bound, $E_{0}$, has been replaced by $E_{g+\frac{1}{2}}$, the energy boundary between groups $g$ and $g+1$. For uniform group widths, the multigroup and boundary fluences are approximately related by

$$
S_{g+\frac{1}{2}} \phi\left(\mu, E_{g+\frac{1}{2}}\right) \cong \frac{S_{g} \phi_{g}(\mu)+S_{g+1} \phi_{g+1}(\mu)}{2 \Delta E} .
$$

Substituting Eq. (9) into Eq. (8) and multiplying by 2, 


$$
\begin{gathered}
2 \frac{S_{g+1} \phi_{g+1}(\mu)}{\Delta E} \cong \int\left\{\frac{1}{2}+\sum_{l=1}^{L}\left(\frac{2 l+1}{2}\right) P_{l}(\mu)\left(\frac{S_{g+1} / \Delta E}{\sigma_{g+1}^{0}-\sigma_{g+1}^{l}}\right)\right. \\
\left.\left[1-e^{-\left(\frac{\sigma_{g+1}^{0}-\sigma_{g+1}^{l}}{S_{g+1}}\right) \Delta E}\right] P_{l}\left(\mu^{\prime}\right)\right\}\left(\frac{S_{g} \phi_{g}(\mu)+S_{g+1} \phi_{g+1}(\mu)}{\Delta E}\right) d \mu^{\prime},
\end{gathered}
$$

where the Legendre expansion has been truncated at order $L$. Multigroup-Legendre cross sections are determined by comparing Eq. (10) with Eq. (7). The total cross section is

$$
\sigma_{t, g}=\frac{2 S_{g}}{\Delta E}
$$

The zero moment scattering cross sections are

$$
\sigma_{g \rightarrow g}^{0}=\sigma_{g \rightarrow g+1}^{0}=\frac{S_{g}}{\Delta E}
$$

and the higher-order self-scatter cross sections are

$$
\sigma_{g \rightarrow g}^{l}=\sigma_{g \rightarrow g}^{0}\left(\frac{S_{g} / \Delta E}{\sigma_{g}^{0}-\sigma_{g}^{l}}\right)\left[1-e^{-\left(\frac{\sigma_{g}^{0}-\sigma_{g}^{l}}{S_{g}}\right) \Delta E}\right]
$$

and the higher-order down-scatter moments are

$$
\sigma_{g \rightarrow g+1}^{l}=\sigma_{g \rightarrow g+1}^{0}\left(\frac{S_{g+1} / \Delta E}{\sigma_{g+1}^{0}-\sigma_{g+1}^{l}}\right)\left[1-e^{-\left(\frac{\sigma_{g+1}^{0}-\sigma_{g+1}^{l}}{S_{g+1}}\right) \Delta E}\right]
$$

To ensure particle balance, an absorption term is added to the lowest-energy group

$$
\sigma_{a, G}=\frac{S_{G}}{\Delta E}
$$

\section{B. Method 2: Linear-continuous differencing}

A slightly different relationship ${ }^{10}$ between the multigroup and boundary fluences results in more accuracy but generates negative cross sections. The multigroup fluence is approximated by

$$
S_{g} \phi_{g}(\mu) \cong \frac{S_{g+\frac{1}{2}} \phi\left(\mu, E_{g+\frac{1}{2}}\right)+S_{g-\frac{1}{2}} \phi\left(\mu, E_{g-\frac{1}{2}}\right)}{2 \Delta E_{g}} .
$$

Substituting Eq. (16) into Eq. (8) results, after some manipulation, in the following cross sections. The total cross section is

$$
\sigma_{t, g}=\frac{2 S_{g}}{\Delta E_{g}}
$$

and the self-scatter cross sections are zero. The zero-moment down scatter cross sections are

$$
\sigma_{g \rightarrow g^{\prime}}^{0}=4(-1)^{g-g^{\prime}-1} \frac{S_{g}}{\Delta E_{g}}
$$


and the higher-order down-scatter moments are

$$
\sigma_{g \rightarrow g^{\prime}}^{l}=\sigma_{g \rightarrow g^{\prime}}^{0}\left(\frac{S_{g^{\prime}} / \Delta E_{g^{\prime}}}{\sigma_{g^{\prime}}^{0}-\sigma_{g^{\prime}}^{n}}\right)\left[1-e^{-\left(\frac{\sigma_{g^{\prime}}^{0}-\sigma_{g^{\prime}}^{l}}{S_{g^{\prime}}}\right) \Delta E_{g^{\prime}}}\right] .
$$

To ensure particle conservation, an absorption is added

$$
\sigma_{a, g}=2(-1)^{G-g} \frac{S_{g}}{\Delta E_{g}}
$$

\section{SUMMARY}

The Goudsmit-Saunderson approach combines the elastic scattering and CSD terms in a single downscatter cross section. By averaging over energy groups, self-scatter and down-scatter cross sections are determined that are much less forward peaked than the true cross sections they represent and are, in certain circumstances, amenable to a low-order Legendre-expansion approximation. We now demonstrate good agreement with experiment and Monte Carlo results for $1-\mathrm{MeV}$ electron beams incident on a variety of materials.

\section{COMPARISON WITH LOCKWOOD DATA}

Lockwood, et al. ${ }^{11}$ have performed a series of careful measurements of energy deposition profiles for 1-MeV electrons normally incident on range-thick materials. The experimental uncertainties are less than a few percent. Fig. 1 shows the excellent agreement between calculation and measurement. Fig. 1 compares dose-depth profiles for electrons on beryllium, aluminum, tantalum, and a composite of aluminum/gold/aluminum.

Electron cross sections were computed with a modified version of the CEPXS ${ }^{5}$ code and the transport was performed with the ONELD ${ }^{5}$ discrete ordinates code. ONELD is designed to accept negative cross sections, so the more-accurate linear-continuous difference method was used to generate the cross sections. Table 1 shows the number of energy groups and Legendre expansion order necessary for accurate solution.

\begin{tabular}{|c|c|c|c|}
\hline Material & Energy Groups & Quadrature Order & Legendre Order \\
\hline beryllium & 40 & 32 & 17 \\
aluminum & 50 & 32 & 11 \\
tantalum & 80 & 32 & 5 \\
al/au/al & 80 & 32 & 15 \\
\hline
\end{tabular}

Table 1: Parameters required for discrete ordinates modeling of Lockwood data. 


\section{COMPARISON WITH MONTE CARLO}

Fig. 2 shows energy-deposition distributions for a $1-\mathrm{MeV}$ electron beam normally incident on a bar of aluminum that is about a half-range thick. The figure compares results from the Monte Carlo code ACCEPT $^{4}$ with those from TWODANT ${ }^{2}$. The agreement is very good. The Monte Carlo results are less-smooth, due to the statistical nature of the solution. The discrete ordinates results exhibit some numerical straggling, which tends to spread out the beam a bit too quickly with penetration, but this is not too serious for this problem.

Table 2 compares runtimes of the Monte Carlo and discrete ordinates solutions. The Monte Carlo results are listed with corresponding statistical uncertainty ranges over the 1,600 cells used to compute the energy-deposition distributons. The timing of the Monte Carlo runs is very much dependent upon the accuracy in the resulting solution, especially off the beam axis where the result is small. The Monte Carlo times are also strongly dependent on the number of cells used to compute the energy-deposition distribution. Times for the TWODANT results are compared with corresponding runs with DORT. The DORT results are not shown, but also agree well with Monte Carlo.

\section{SUGGESTIONS FOR FUTURE WORK}

The SMART multigroup-Legendre cross sections should work well in adjoint calculations, as they do in forward calculations, although this has not been thoroughly tested as yet. The cross sections should also be effective in multigroup Monte Carlo calculations.

The SMART multigroup-Legendre cross sections have been demonstrated to work over a wide variety of problems. However, there are some problems for which the cross sections have not worked so well. For high-energy electron beams on water (of importance in radiation oncology studies) a very high-order Legendre expansion is required. For these types of problems some type of first-collision source technique may prove effective ${ }^{12}$. For high-energy electron beams on high- $Z$ materials a large number of energy groups is required to reduce numerical straggling. Allowing for non-uniform electron energy group widths would improve the modeling, in some cases.

This work was supported by the U.S. Departement of Energy Contract DE-AC04-94-AL85000.

\begin{tabular}{|c|c|c|c|}
\hline Code & Method & $\begin{array}{c}\text { Fractional } \\
\text { Standard } \\
\text { Deviation }\end{array}$ & $\begin{array}{c}\text { Single-Processor } \\
\text { SPARC1000 CPU } \\
\text { (minutes) }\end{array}$ \\
\hline ACCEPT & Monte Carlo & 0.01 to 0.24 & 38 \\
ACCEPT & Monte Carlo & 0.0 to 0.05 & 620 \\
DORT & Discrete Ordinates & na & 41 \\
TWODANT & Discrete Ordinates & na & 88 \\
\hline
\end{tabular}

Table 2: Timing comparisons: discrete ordinates vs. Monte Carlo. 


\section{REFERENCES}

1. W. A. Rhoades and R. L. Childs, "An Updated Version of the DOT 4 One- and TwoDimensional Neutron/Photon Transport Code," Oak Ridge National Laboratory Report, ORNL5851 (1982).

2. R. E. Alcouffe, et al., "User's Guide for TWODANT: A Code Package for Two-Dimensional, Diffusion-Accelerated, Neutral-Particle Transport," Los Alamos National Laboratory Report, LA-10049-M, Rev. 1 (1984).

3. W. A. Rhoades and R. L. Childs, "The TORT Three-Dimensional Discrete Ordinates Neutron/Photon Transport Code," Oak Ridge National Laboratory Report, ORNL-6268 (1987).

4. J. A. Halbleib, R. P. Kensek, G. D. Valdez, S. M. Seltzer and M. J. Berger, "ITS: The Integrated TIGER Series of Coupled Electron/Photon Monte Carlo Transport Codes - Version 3.0," IEEE Trans. Nucl. Sci., 39, 1025 (1992).

5. L. J. Lorence, Jr., J. E. Morel, and G. D. Valdez, "Physics Guide to CEPXS: A Multigroup Coupled Electron-Photon Cross-Section Generating Code," Sandia National Laboratories report, SAND89-1989 (1989).

6. W. L. Filippone, "The Theory and Application of SMART Electron Scattering Matrices," Nucl. Sci. Eng., 99, 232 (1988).

7. G. I. Bell and S. Glasstone, Nuclear Reactor Theory, p. 13, Van Nostrand Reinhold Company, New York (1970).

8. J. E. Morel, "Fokker-Planck Calculations Using Standard Discrete Ordinates Transport Codes," Nucl. Sci. Eng., 79, 340 (1981).

9. S. Goudsmit and J. L. Saunderson, "Multiple Scattering of Electrons," Phys. Rev. 57, 24 (1940).

10. J. E. Morel, "Multigroup Legendre Coefficients for the Diamond Difference Continuous Slowing Down Operator," Nucl. Sci. Eng., 91, 324 (1985).

11. G. J. Lockwood, L. E. Ruggles, G. H. Miller, and J. A. Halbleib, "Calorimetric Measurement of Electron Energy Deposition in Extended Media - Theory vs Experiment," Sandia National Laboratories report, SAND79-0414 (1979).

12. W. L. Filippone, et al., "An Extended First Collision Source Method for Electron Beam Source Problems," Nucl. Sci. Eng., 112, 1 (1992). 

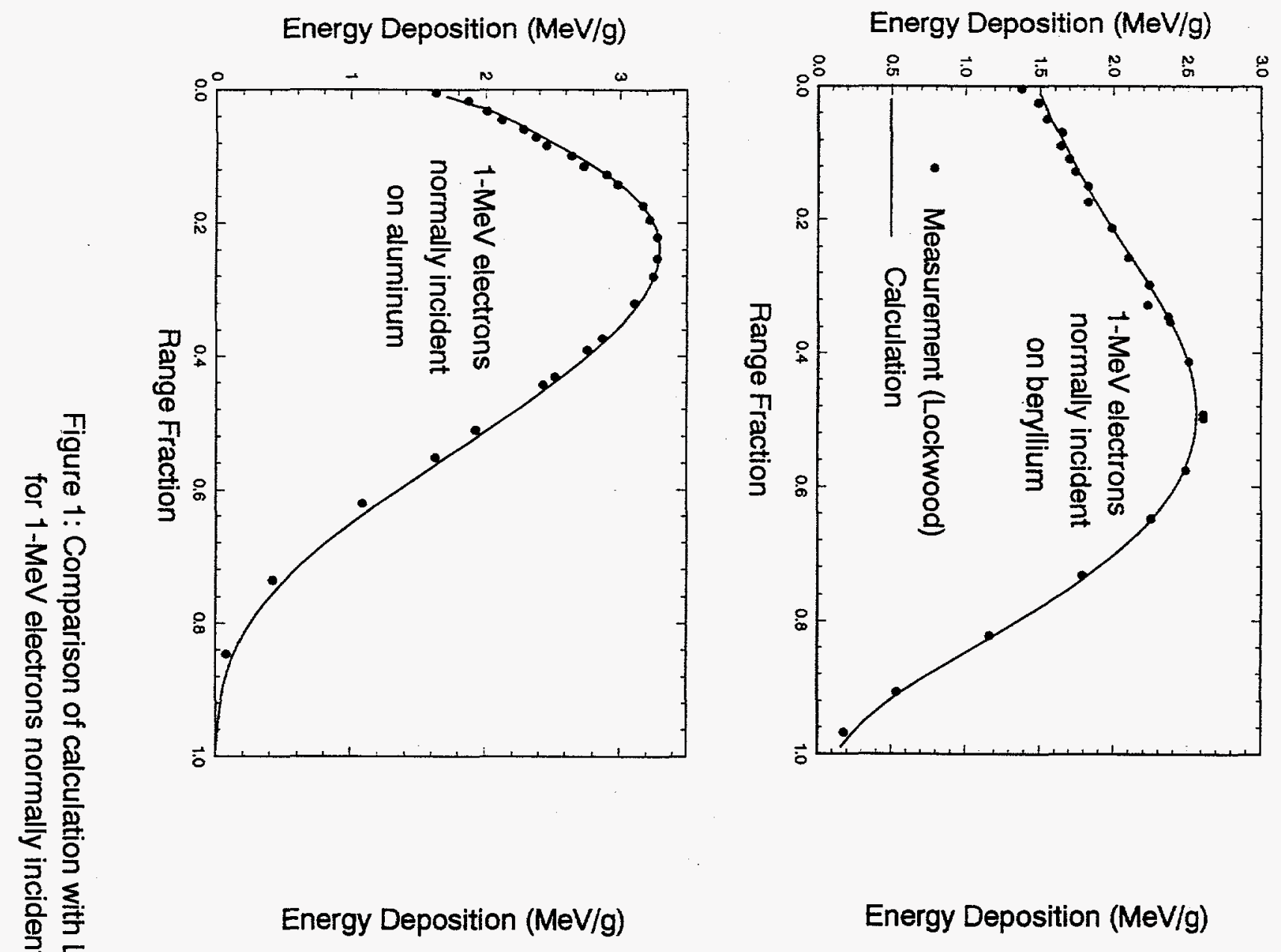

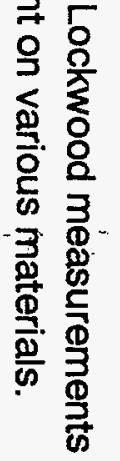
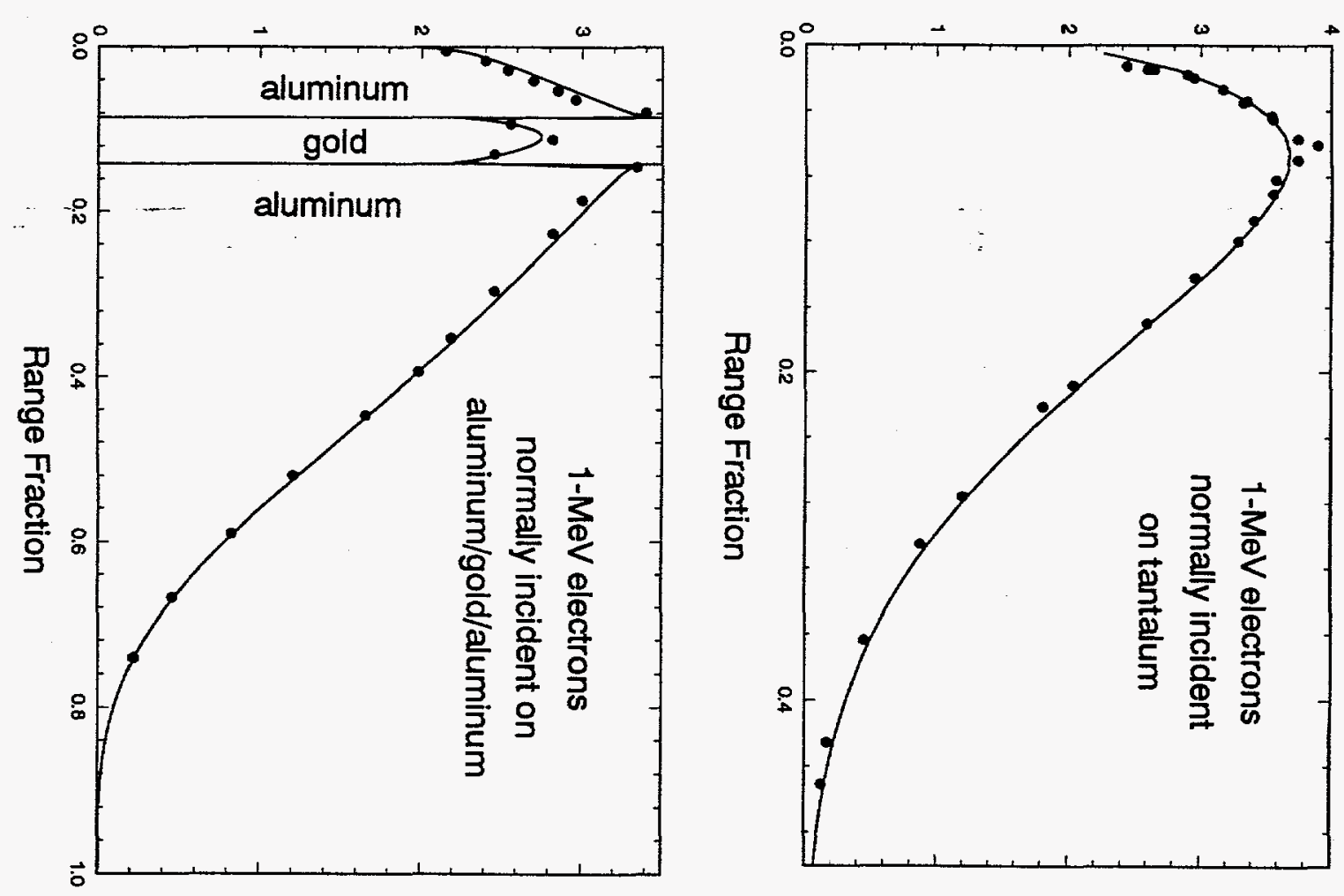


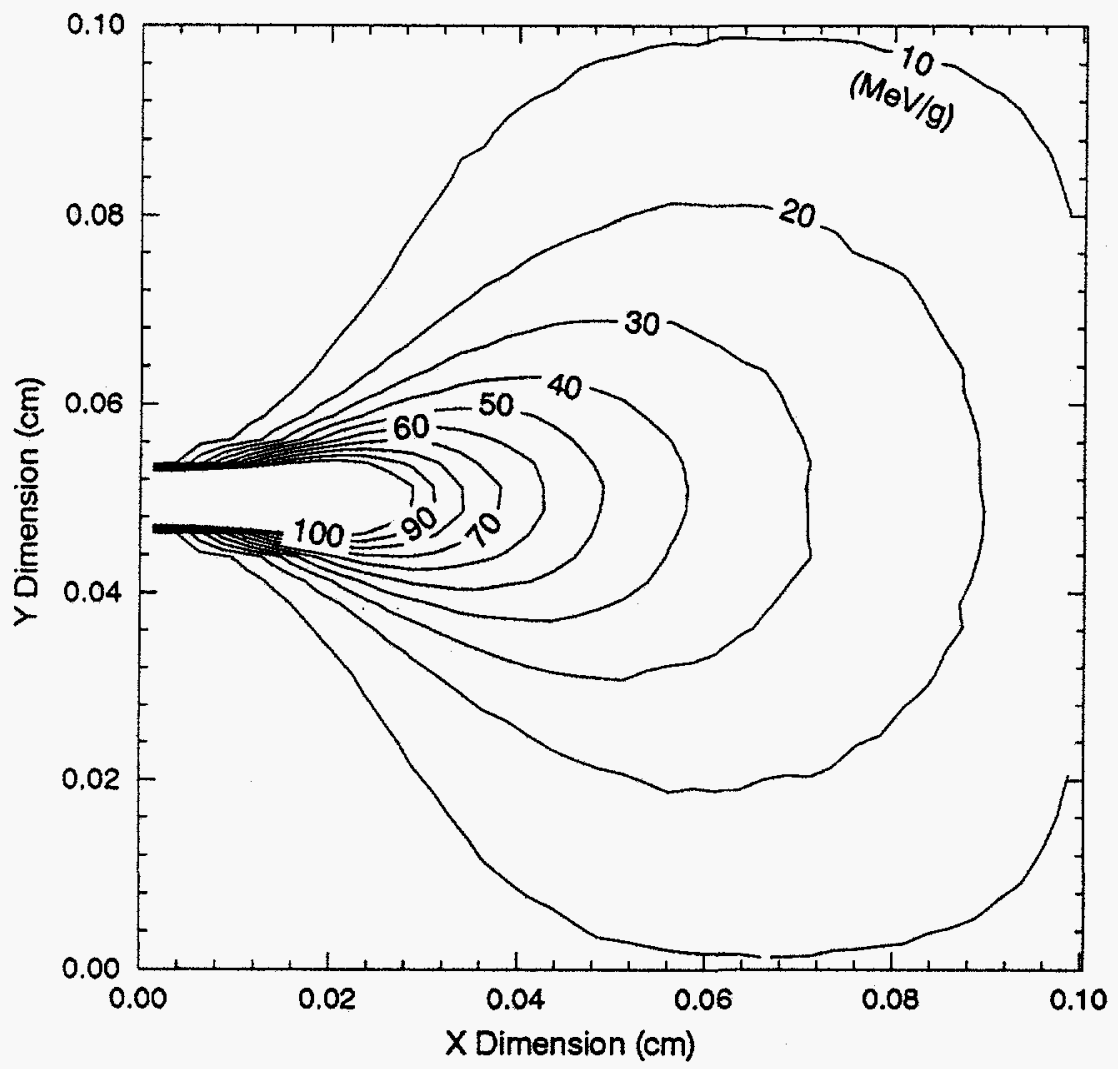

ACCEPT (Monte Carlo)

Energy Deposition

Contours (MeV/g)

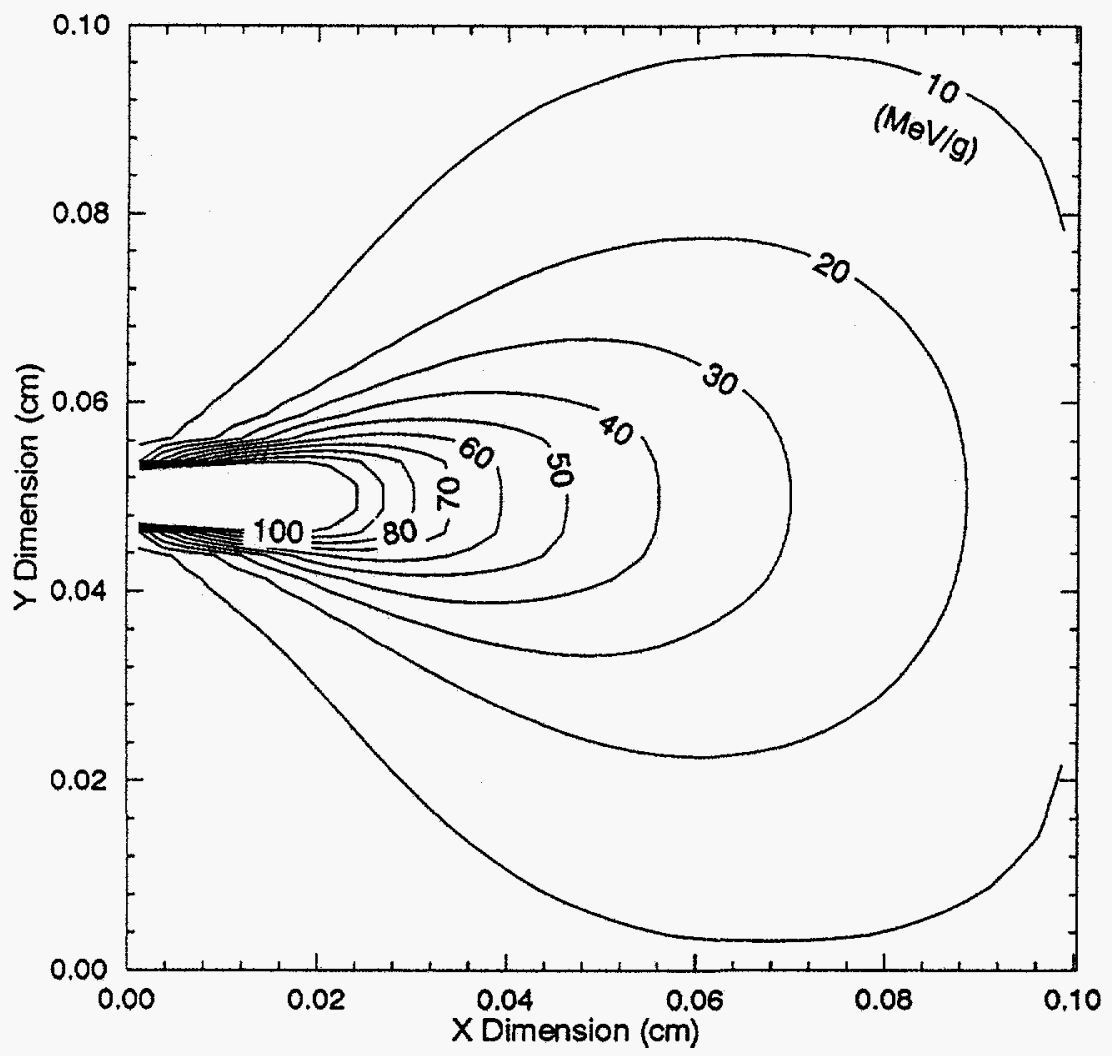

CEPXS/TWODANT

(Discrete Ordinates)

Energy Deposition

Contours (MeV/g)

Figure 2: Comparison of ITS Monte Carlo and CEPXS/TWODANT calculations for $1-\mathrm{MeV}$ electron beam normally incident on aluminum bar. 


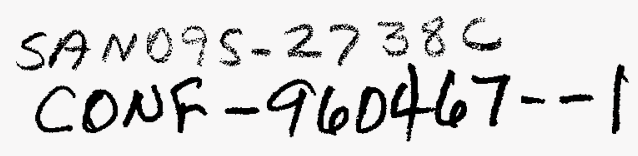

\title{
MULTIDIMENSIONAL ELECTRON-PHOTON TRANSPORT WITH STANDARD DISCRETE ORDINATES CODES
}

Clifton R. Drumm

Sandia National Laboratories

MS 1166, PO Box 8500

Albuquerque, New Mexico 87185-1166

(505) 845-7953

\begin{abstract}
A method is described for generating electron cross sections that are compatible with standard discrete ordinates codes without modification. There are many advantages of using an established discrete ordinates solver, e.g. immediately available adjoint capability. Coupled electron-photon transport capability is needed for many applications, including the modeling of the response of electronics components to space and man-made radiation environments. The cross sections have been successfully used in the DORT, TWODANT and TORT discrete ordinates codes. The cross sections are shown to provide accurate and efficient solutions to certain multidimensional electronphoton transport problems.
\end{abstract}

\section{INTRODUCTION}

This article describes the extension of neutral-particle discrete ordinates codes for electron transport applications. Electron cross sections are computed that are compatible with standard discrete ordinates codes without modification. To the extent that these cross sections approximate the physics of the electron interactions, neutral-particle codes, such as DORT ${ }^{1}$, TWODANT ${ }^{2}$ and TORT $^{3}$, are able to model electron and coupled photon-electron-positron transport.

The advantages of such an approach are many. Adjoint capability is immediately available. Standard codes have undergone extensive benchmarking and quality assurance (QA). From a user standpoint, using the same transport code to model neutron, photon, and charged particle transport is efficient. Acceleration techniques that have been developed for neutral-particle transport apply to a certain extent to charged-particle transport.

There are also some problems involved with using a neutral-particle code for charged-particle transport. Production discrete ordinates codes are unable to model $\delta$-function downscattering, which is inherent in the continuous slowing down approximation (CSDA) describing charged particle transport. Furthermore, because the charged-particle interaction cross sections are generally much larger than their neutral-particle counterparts, traditional first-collision source techniques 